\title{
Successful Implantation and Live Birth Following Autologous Platelet-rich Plasma Treatment for a Patient with Recurrent Implantation Failure and Chronic Endometritis
}

\author{
KONSTANTINOS SFAKIANOUDIS ${ }^{1 *}$, MARA SIMOPOULOU ${ }^{2 *}$, NIKOLAOS NITSOS $^{3}$, LEANDROS LAZAROS $^{4}$, \\ ANNA RAPANI ${ }^{2}$, AGNI PANTOU ${ }^{1}$, MICHAEL KOUTSILIERIS ${ }^{2}$, YORGOS NIKAS ${ }^{5}$ and KONSTANTINOS PANTOS ${ }^{1}$ \\ ${ }^{1}$ Reproductive Medicine Unit, Genesis Athens Clinic, Athens, Greece; \\ ${ }^{2}$ Department of Physiology, School of Medicine, National and Kapodistrian University of Athens, Athens, Greece; \\ ${ }^{3}$ Genesis Athens Clinic, Microbiology-Biochemical Department, Athens, Greece; \\ ${ }^{4}$ Genesis Genoma Lab, Molecular Genetics, Athens, Greece; \\ ${ }^{5}$ Athens Innovative Microscopy, Athens, Greece
}

\begin{abstract}
Background/Aim: Patients diagnosed with chronic endometritis (CE) may fail to respond to standard antibiotic treatment. The driver behind the approach reported here was the imperative need for alternative therapeutic solutions. Case Report: This case report presents a woman with CE and premature ovarian insufficiency having experienced repeated implantation failures following donated embryo transfers. The patient was diagnosed with CE through hysteroscopy, microbiological analysis and scanning electron microscopy. Following the suggested antibiotic treatment, she underwent a new embryo transfer, but with subsequent pregnancy loss. Following a second antibiotic scheme, all diagnostic procedures certified the persistence of CE. The patient underwent autologous, intrauterine platelet-rich plasma treatment and a subsequent embryo transfer. The diagnostic procedures indicated no signs of CE, while the embryo transfer resulted in a twin pregnancy and birth. Conclusion: Platelet-rich plasma may be employed as a first-line CE treatment, especially for patients who fail to respond to conventional antibiotic schemes.
\end{abstract}

This article is freely accessible online.

*These Authors contributed equally to this study.

Correspondence to: Mara Simopoulou, Assistant Professor of Physiology, Clinical Embryologist/Geneticist, Department of Physiology-Department of Obstetrics and Gynecology, Medical School, National and Kapodistrian University of Athens, 75, Mikras Asias, 11527 Athens, Greece. Tel/Fax: +30 2107462592, +30 2107462571, e-mail: marasimopoulou@hotmail.com

Key Words: Chronic endometritis, implantation failure, inflammation, IVF, pregnancy, PRP.
Chronic endometritis (CE) is a persistent inflammatory condition of the inner lining of the uterine cavity. It is characterized by infiltration of plasma cells and lymphocytes present in the endometrial stroma, increased cell density of the stroma, asynchronous maturation of epithelial cells and stromal fibroblasts, as well as by superficial mucosal oedema. The inflammation is often asymptomatic or oligosymptomatic with abnormal uterine bleeding and vaginal discharge, lower abdominal pain, fever, leukorrhea and menstrual dysfunction (1). A growing body of evidence points to patients with endometriosis presenting more frequently with $\mathrm{CE}$ further associated with infertility of unknown aetiology, unexplained repeat pregnancy losses and repeat embryo implantation failures following in vitro fertilization (IVF) treatments (2-5). Endometrial receptivity in $\mathrm{CE}$ can be defined as dysfunctional, since the local expression of numerous genes encoding cytokines, chemokines, apoptotic proteins and adhesion molecules is dysregulated (6-8). On the subject of CE and implantation failure, decidualization and the mechanism of its modification may provide an explanation. The process of decidualization takes place in endometrial stromal cells. Decidua differentiates to maintain pregnancy, while it produces growth factors, hormones and cytokines (9). Its role may be pivotal in regulating trophoblast invasion and the challenge presented by the maternal immune system $(10,11)$. Therefore, the association between CE and implantation failure may be provided given the role of the decidua on implantation and the fact that CE appears to modify the process of decidualization (9).

$\mathrm{CE}$ is considered to be attributed to ascending infection caused by organisms of the indigenous vaginal flora, sexually-transmitted infections, and tuberculosis, as well as through medical procedures that enable a pathway for bacteria to enter the uterus through the cervix. In the 
endometrium in cases of $\mathrm{CE}$, the most commonly detected microorganisms are Streptococcus species, Corynebacterium, Escherichia coli, Enterococcus faecalis, Mycoplasma genitalium, Bacteroides bivius and Ureaplasma urealyticum (12, 13), while earlier studies by Haggerty et al. also supported the implication of Neisseria gonorrhoeae and Chlamydia trachomatis in CE onset (14). Finally, fungal agents have been reported rarely as being responsible for infectious causes of CE (15). Immune response due to the presence of microbial infections is described to be relatively atypical of the mucosal tissue environment, causing infiltration of circulating B-cells in the endometrial stromal and glandular epithelial areas. Endometrial B-cells are normally located within the basal layer, while their accumulation in the functional layer is caused by proinflammatory molecules that are abnormally expressed (8).

Oral antimicrobial regimes are considered to be the gold standard in the treatment of CE. Although antimicrobial agents eliminate endometrial stromal plasmacytes, the endometrial/intrauterine microbial profile alterations of patients with CE remain unclear. A wide spectrum of antibiotic treatments has been proposed for $\mathrm{CE}$ such as doxycycline, ciprofloxacin, metronidazole, ofloxacin, amoxicillin, clavulanate, josamycin and minocycline. However, certain CE cases do not respond satisfactorily to these widely applied treatments, driving research towards addressing the need for alternative options when managing these cases (2).

Platelet-rich plasma (PRP) use in the treatment of various medical cases, including osteoarthritis and tendonitis, has been introduced due to its antimicrobial and antiinflammatory properties $(16,17)$. In light of the above findings, we sought to explore whether autologous, intrauterine PRP treatment might represent a treatment for CE. PRP is prepared by peripheral blood withdrawal following centrifugation in order to achieve a high concentration of platelets (18). Platelets carry more than 800 proteins, namely cytokines, hormones and chemoattractants of stem cells, macrophages and neutrophils, responsible for various post-translational modifications of nearly 1,500 bioactive factors (19). Platelets also carry numerous growth factors of great significance due to their ability to repair tissue as well as their mitogenic, chemotactic, neovascular and anti-inflammatory effects (20). These factors include: vascular endothelial growth factor (VEGF), platelet-derived growth factor (PDGF), transforming growth factor beta (TGF- $\beta$ ), keratinocyte growth factor (KGF), connective tissue growth factor (CTGF), basic fibroblast growth factor (bFGF), insulin-like growth factor 1 (IGF-I), epidermal growth factor (EGF) and 2 (IGF-II) and hepatocyte growth factor (HGF), which are released following alpha granule activation by native or exogenous molecules, including thrombin, collagen, magnesium and calcium chloride. The aforementioned proteins play fundamental roles regarding inflammation control, tissue regeneration, angiogenesis activation, anabolism increase as well as cell migration, differentiation and proliferation (21-23). Further to the above, supplementing cell cultures with PRP has been suggested by various research groups due to its ability to induce cell proliferation (24-26).

\section{Case Report}

In the current study, a 35-year-old woman, with premature ovarian insufficiency and a history of six failed donated embryo transfers, was referred to our clinic for assisted reproduction. The patient's detailed medical history was recorded and blood samples were taken. Molecular testing excluded thrombophilia. Thorough assessment of metabolism and hormonal function including glucose tolerance test, prolactin and thyroid function tests were also performed.

Hysteroscopy was performed during the luteal phase of the menstrual cycle of the patient, employing a lens-based $3 \mathrm{~mm}$ OD mini-telescope, 1058 angle of visual field equipped with a $3.5 \mathrm{~mm}$ OD single-flow diagnostic sheath. The required uterine cavity distention was performed with the use of saline, while a $300 \mathrm{~W}$ light source with a xenon bulb, a digital camera and a 21-inch video screen were employed. The cavity was panoramically evaluated assessing the endometrial mucosa. The presence of micropolyps, polypoid endometrium, stromal oedema and diffuse hyperaemia was taken into account and $\mathrm{CE}$ was the established diagnosis. A $3 \mathrm{~mm}$ Novak curette connected to a 20-ml syringe was employed in order to perform biopsy of the endometrium and analyse infectious agents. Contamination risk was controlled employing a vaginal speculum and by cleaning the external uterine ostium with iodine solution. Novak cannula application was performed under visual control, aiming to avoid contact with vaginal walls. Endometrial samples were placed into two 2-ml aliquots of saline for microbiological and scanning electron microscopy (SEM) analyses. Literature does not report on SEM analysis as a diagnostic tool with regards to $\mathrm{CE}$. In the context of this work, SEM evaluation provided practitioners with supplementary data pertaining to the endometrium's overall condition. The microbiological analysis evidenced the presence of Candida albicans. The second aliquot was placed in neutral formalin and later embedded in paraffin for SEM examination. SEM revealed increased plasma cell concentrations and mycoplasma accumulations in the endometrial stroma of the patient, confirming the hysteroscopic and microbiological findings.

Based on the infectious agent detected, an appropriate antimycotic treatment was prescribed. Specifically, the patient received $800 \mathrm{mg}$ fluconazole daily for 7 days according to the recommendations published by the Infectious Diseases Society of America (27). Following the antimycotic therapy and suitable hormonal treatment, the patient underwent 
A



C



B

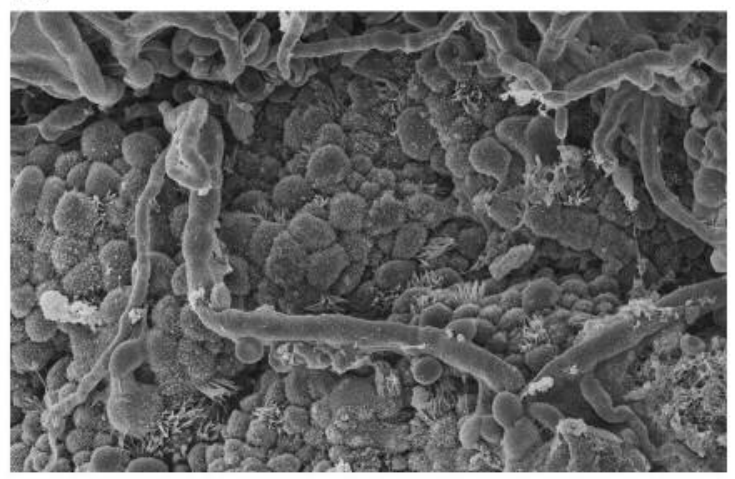

D

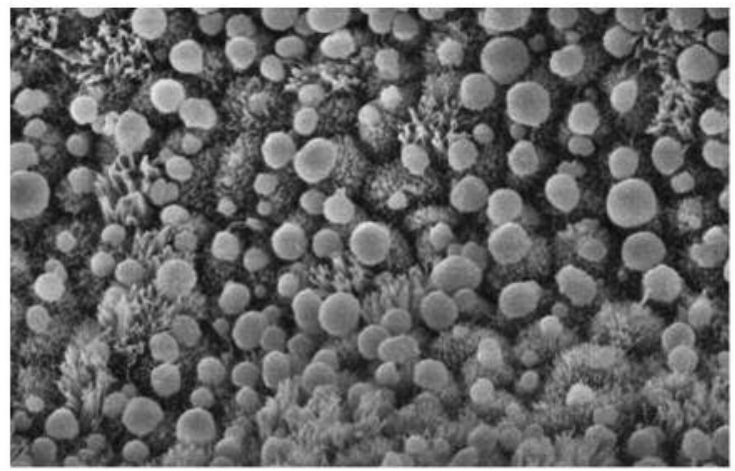

Figure 1. Scanning electron microscopy analysis of the patient's inner endometrial lining at the luteal phase of the menstrual cycle after the second antimycotic scheme $(A$ and $B)$ and after platelet-rich plasma $(P R P)$ treatment $(C$ and $D)$. A: Two gland orifices of the infected endometrial epithelium are visible, containing mucus and blood cells. Clusters of mucus are also present on the epithelium surface (magnification $\times 750$ ). B: Fungal spores, red blood cells and mucus in endometrial biopsy. The hyphae are in contact with the epithelial cells, fusing with some of them (magnification $\times 2,700)$. C: Normal endometrial epithelium morphology was apparent following PRP treatment (magnification $\times 750$ ). D: Presence of pinopodes in the cured endometrial epithelium following PRP treatment (magnification $\times 2,700$ ).

transfer of two donated blastocysts. Blastocyst grading was performed according to the criteria of Gardner et al. (28). The two blastocysts were graded as 5AA and pregnancy was confirmed 2 weeks following embryo transfer by human chorionic gonadotropin $(\beta-\mathrm{hCG})$ quantification. Although $\beta$-hCG was increasing normally, abnormal bleeding and subsequent pregnancy loss took place 20 days post-embryo transfer. A second administration including the same antimycotic scheme as previously employed, was performed for the same period of time. Following the above treatment, as well as appropriate endometrial preparation, the patient underwent a second hysteroscopy at the luteal phase of the menstrual cycle for CE signs, while endometrial samples for SEM and culture were collected. All analyses confirmed the persistence of $\mathrm{CE}$, while SEM revealed increased inflammation (Figure 1A and B).

Taking into account the effectiveness of PRP on different inflammation types, we performed an autologous, intrauterine PRP treatment. In accordance with the Helsinki declaration, the present study protocol was approved by the Hospital Ethics
Committee (reference number: 11/8-1-2018). Following informed consent for the treatment from the couple, preparation of the autologous PRP was performed during the follicular phase of the cycle employing RegenACR ${ }^{\circledR}$-C Kit (Regen Laboratory, Le Mont-sur-Lausanne, Switzerland) following instructions provided by the manufacturer. The volume of peripheral blood required to yield approximately $2.5 \mathrm{ml}$ of prepared PRP for infusion for this patient was $25 \mathrm{ml}$. The initial density of platelets in the peripheral blood sample was $250,000 / \mu \mathrm{l}$ while that of the prepared PRP was $900,000 / \mu 1$. Per our protocol, prepared PRP was allowed to be stored for $1 \mathrm{~h}$ at $4^{\circ} \mathrm{C}$. However, for this case it was administered immediately following preparation. The endometrial infusion was performed employing transvaginal ultrasound guidance and use of an appropriate catheter (Gynetics Medical Products N.V., Lommel, Belgium). The volume of PRP infused into the uterine cavity was approximately $2.5 \mathrm{ml}$.

Following PRP treatment and endometrial preparation, a third hysteroscopy was performed at the follicular stage of the subsequent menstrual cycle, while an endometrial sample 
was extracted at the respective luteal phase. Subsequent hysteroscopy, microbiological analysis and SEM (Figure 1C and D) showed no signs of CE.

Our patient underwent another embryo transfer of two donated, blastocysts graded as $5 \mathrm{BB}$ and $5 \mathrm{BC}$ at the next menstrual cycle, which resulted in a twin pregnancy. Four weeks following a positive $\beta$-hCG pregnancy test, clinical pregnancy was confirmed by observing foetal cardiac activity on transvaginal ultrasound. The babies were delivered at the 36 th week of gestation and weighed was $2.28 \mathrm{~kg}$ and $2.18 \mathrm{~kg}$.

\section{Discussion}

This case report uniquely presents a patient with $\mathrm{CE}$ who failed to respond to the standard approach of antibiotic regime and who was successfully treated for CE following an autologous intrauterine PRP treatment. CE in this case was associated with infertility due to premature ovarian insufficiency and recurrent implantation failure with donated embryos. This case was effectively managed employing autologous intrauterine PRP, which in turn allowed successful treatment of $\mathrm{CE}$ and enabled subsequent implantation of transferred embryos leading to live birth of twins. Although the development of medically assisted reproduction technology has presented a valid solution to the issues of female infertility, a considerable proportion of infertile women still fail to be successfully managed with respect to achieving a pregnancy. Recurrent implantation failures and pregnancy losses have either maternal or foetal aetiology (29). Among the most frequent maternal causes are thrombophilia, abnormal uterine anatomy, impaired endometrial receptivity, as well as various immunological factors (30). Unfortunately, the above aggravating factors are usually diagnosed $a$ posteriori. CE, which has direct effects on IVF success and pregnancy maintenance, is often asymptomatic and undetectable by ultrasound or hysterosalpingography.

Elimination of inflammation of the uterine stroma constitutes the basis of CE therapy. Various mechanisms may serve this aim, such as the augmented apoptosis of proinflammatory cells (31), the diminished expression of a considerable list of factors, including prostaglandins, proinflammatory cytokines (IL-1, TNF $\alpha$ ) and matrix metalloproteinases, as well the heightened expression of antiinflammatory molecules, including interleukin 1 receptor antagonist and tumor necrosis factor receptor (TNFR). To add to this list, the synthesis and release of antiinflammatory cytokines (IL-4, IL-10) (32), TGF- $\beta$ (33) and lipoxins (34) contribute to managing successfully uterine stroma inflammation along the therapeutic basis of CE. Furthermore, the cleavage of chemokines with matrix metalloproteinases has been found to trigger the production of anti-inflammatory factors, while the increased cell survival in the inflammation regions caused by interacting with the extracellular matrix frequently contributes to terminating the inflammation (35).

Intrauterine PRP treatment may activate many of the above processes. Applying platelet-derived factors into areas of damaged tissues could endorse healing renewal and regeneration (36-38). The bioactive nature of these factors consisting of coagulation factors, proteins with antibacterial and fungicidal effects and membrane glycoproteins directly and proportionally affects interleukins and chemokines synthesis, reported to enable management of inflammation symptoms (39). Further on, delineating the healing pathway triggered by PRP application through tissue homeostasis, stimulation is ensured by exposure to dense granule-derived factors, such as adenoside diphosphate, adenoside triphosphate, serotonin, histamine, dopamine and calcium ions as reported by Zhu et al. (39), whereas alpha granule-released growth factors are profoundly crucial in achieving wound healing and tissue regeneration (40). A recent study, examining mares with chronic degenerative endometritis, has shown that uterine PRP infusion as an immunomodulator of the inflammatory response, can modulate the local inflammatory response by reducing the number of polymorphonuclear neutrophils into the uterine lumen and preventing intrauterine fluid retention (20). In addition, the intrauterine PRP treatment has been showed to promote endometrial growth improving the pregnancy outcome of patients presenting with thin endometrium due to the growth factors PRP consists of such as vascular endothelial growth factor, platelet-derived growth factor, transforming growth factor beta, insulin-like growth factor, epidermal growth and fibroblast growth factor 2, factor that stimulate the healing process (41-43). In vitro results of a very interesting study on the potential of PRP to act as promoter of follicular development strengthen the hypothesis that PRP is a most dynamic source of growth factors effectively supporting viability, regeneration and growth (44). In light of the above, it may be extrapolated that autologous, intrauterine PRP treatment on this patient probably modulated the aberrant inflammatory processes of $\mathrm{CE}$ while promoting healing of the endometrial stroma.

The underlying mechanisms through which PRP acts as a modulator alleviating inflammation are further strengthened by in vitro studies of PRP treatment on animal models $(20,45)$. Proliferation as well as gene expression of endometrial cells have already been demonstrated and confirmed by in vitro bovine studies. However, the mechanism by which this gene regulation is achieved deserves to be further investigated. Although, the molecular mechanisms still belong in the grey zone, it is believed that the PRP-derived growth factors play the leading role. A study by Marini et al. demonstrated that the expression of $c-M Y C$ gene was up-regulated in endometrial cells which were cultured with PRP, in comparison to the expression levels found in untreated cells (45). $c-M Y C$ is involved in cell 
proliferation and growth, and is activated by EGF, a component of PRP. This is one representative of many examples of the effects of PRP, demonstrating its effectiveness in reducing gene expression of pro-inflammatory factors such as interleukin $1 \beta$, interleukin 8 , prostaglandin-endoperoxide synthase 2 , and inducible nitric oxide synthetase, revealing its promising prospectives in in vivo regenerative therapy in endometritis (45). El-Sharkawy et al. reported that treatment with PRP increased the release of various growth factors such as insulin-like growth factor-I, fibroblast growth factor, epidermal growth factor and transforming factor $\beta$ (46). The same study of the response of the injured area highlighted the presence of vascular endothelial growth factor as well as a local increase in lipid molecules (46). In addition, PRP also increased chemokine expression, the importance of which relates to the fact that these molecules can inhibit the relocation of leukocytes around the tissue affected (47). An in vitro study on mares by Reghini et al. reports similarly solid data of the ability of PRP to reduce the influx of polymorphonuclear neutrophils (20).

Published data indicate that PRP infusion may be employed for the distinct category of women presenting with poor ovarian reserve or response. The use of PRP in the reproductive context was pioneered by our team and cited by Sills and colleagues (48). Further to that, two case series have been published reporting on ovarian rejuvenation following intraovarian PRP infusion in women characterized as either peri-menopausal (49), or of poor ovarian response (50). Regarding this case report, what should be further highlighted as strengthening the cause and effect association between PRP and treatment of CE enabling pregnancy and live birth is the fact that the quality of the donated embryos that led to a miscarriage was in fact superior when compared to the quality of the embryos that actually resulted in live birth following PRP treatment.

This study uniquely documents that autologous intrauterine PRP treatment holds the potential of being employed as a successful therapeutic tool for CE. Verifying our findings in larger patient groups through randomized controlled studies would strengthen this finding and secure the role of PRP as a successful therapeutic means for patients with $\mathrm{CE}$, especially for those that fail to respond to conventional antibiotic schemes. The current approach was successful on two intertwined levels as infertility was addressed and treated following PRP treatment of CE, allowing for the successful implantation of two donated embryos leading to subsequent clinical pregnancies and live births.

\section{References}

1 Kitaya K, Matsubayashi H, Yamaguchi K, Nishiyama R, Takaya Y, Ishikawa T,Yasuo T and Yamada H: Chronic endometritis: potential cause of infertility and obstetric and neonatal complications. Am J Reprod Immunol 75: 13-22, 2016.
2 Kitaya K, Matsubayashi H, Takaya Y, Nishiyama R, Yamaguchi K, Takeuchi $\mathrm{T}$ and Ishikawa T: Live birth rate following oral antibiotic treatment for chronic endometritis in infertile women with repeated implantation failure. Am J Reprod Immunol 78: e12719, 2017.

3 Johnston-MacAnanny EB, Hartnett J, Engmann LL, Nulsen JC, Sanders MM and Benadiva CA: Chronic endometritis is a frequent finding in women with recurrent implantation failure after in vitro fertilization. Fertil Steril 93: 437-441, 2010.

4 McQueen DB, Bernardi LA and Stephenson MD: Chronic endometritis in women with recurrent early pregnancy loss and/or fetal demise. Fertil Steril 101: 1026-1030, 2014.

5 Takebayashi A, Kimura F, Kishi Y, Ishida M, Takahashi A, Yamanaka A, Takahashi K and Suginami H: The Association between Endometriosis and chronic endometritis. PLoS One 9: e88354, 2014.

6 Kitaya K, Tada Y, Hayashi T, Taguchi S, Funabiki M and Nakamura Y: Comprehensive endometrial immunoglobulin subclass analysis in infertile women suffering from repeated implantation failure with or without chronic endometritis. Am J Reprod Immunol 72: 386-391, 2014.

7 Di Pietro C, Cicinelli E, Guglielmino MR, Ragusa M, Farina M, Palumbo MA and Cianci A: Altered transcriptional regulation of cytokines, growth factors, and apoptotic proteins in the endometrium of infertile women with chronic endometritis. Am J Reprod Immunol 69: 509-517, 2013.

8 Kitaya $\mathrm{K}$ and Yasuo T: Aberrant expression of selectin E, CXCL1, and CXCL13 in chronic endometritis. Mod Pathol 23: 1136-1146, 2010.

9 Wu D, Kimura F, Zheng L, Ishida M, Niwa Y, Hirata K, Takebayashi A, Takashima A, Takahashi K, Kushima R, Zhang $\mathrm{G}$ and Murakami T: Chronic endometritis modifies decidualization in human endometrial stromal cells. Reprod Biol Endocrinol 15: 16, 2017.

10 Sharma S, Godbole G and Modi D: Decidual control of trophoblast invasion. Am J Reprod Immunol 75: 341-350, 2016.

11 Vinketova K, Mourdjeva M and Oreshkova T: Human decidual stromal cells as a component of the implantation niche and a modulator of maternal immunity. J Pregnancy 2016: 8689436, 2016.

12 Cicinelli E, De Ziegler D, Nicoletti R, Colafiglio G, Saliani N, Resta L, Rizzi D and De Vito D: Chronic endometritis: correlation among hysteroscopic, histologic, and bacteriologic findings in a prospective trial with 2190 consecutive office hysteroscopies. Fertil Steril 89: 677-684, 2008.

13 Cicinelli E, Matteo M, Tinelli R, Lepera A, Alfonso R, Indraccolo U, Marrocchella S, Greco P and Resta L: Prevalence of chronic endometritis in repeated unexplained implantation failure and the IVF success rate after antibiotic therapy. Hum Reprod Oxf Engl 30: 323-330, 2015.

14 Haggerty CL, Hillier SL, Bass DC and Ness RB: PID Evaluation and Clinical Health study investigators. Bacterial vaginosis and anaerobic bacteria are associated with endometritis. Clin Infect Dis 39: 990-995, 2004.

15 Rodriguez M, Okagaki T and Richart RM: Mycotic endometritis due to Candida: a case report. Obstet Gynecol 39: 292-294, 1972.

16 Bielecki TM, Gazdzik TS, Arendt J, Szczepanski T, Król W and Wielkoszynski T: Antibacterial effect of autologous platelet gel enriched with growth factors and other active substances: an in vitro study. J Bone Joint Surg Br 89: 417-420, 2007. 
17 Woodall J, Tucci M, Mishra A and Benghuzzi H: Cellular effects of platelet rich plasma: a study on HL-60 macrophage-like cells. Biomed Sci Instrum 43: 266-271, 2007.

18 Marx RE: Platelet-rich plasma: evidence to support its use. J Oral Maxillofac Surg 62: 489-496, 2004.

19 Qureshi AH, Chaoji V, Maiguel D, Faridi MH, Barth CJ, Salem SM, Singhal M, Stoub D, Krastins B, Ogihara M, Zaki MJ, Gupta V and Gregson A: Proteomic and phospho-proteomic profile of human platelets in basal, resting state: Insights into Integrin Signaling. PLoS One 4: e7627, 2009.

20 Reghini MFS, Ramires Neto C, Segabinazzi LG, Castro Chaves MMB, Dell'Aqua C de PF, Bussiere MCC, Dell'Aqua JA, Papa FO and Alvarenga MA: Inflammatory response in chronic degenerative endometritis mares treated with platelet-rich plasma. Theriogenology 86: 516-522, 2016.

21 Stiles CD: The molecular biology of platelet-derived growth factor. Cell 33: 653-655, 1983.

22 Pietrzak WS and Eppley BL: Platelet rich plasma: biology and new technology. J Craniofac Surg 16: 1043-1054, 2005.

23 Borrione P, Gianfrancesco AD, Pereira MT, Pigozzi F: Plateletrich plasma in muscle healing. Am J Phys Med Rehabil 89: 854$861,2010$.

24 Akeda K, An HS, Okuma M, Attawia M, Miyamoto K, Thonar EJ-MA, Lenz ME, Sah RL and Masuda K: Platelet-rich plasma stimulates porcine articular chondrocyte proliferation and matrix biosynthesis. Osteoarthritis Cartilage 14: 1272$1280,2006$.

25 Duan J, Kuang W, Tan J, Li H, Zhang Y, Hirotaka K and Tadashi $\mathrm{K}$ : Differential effects of platelet rich plasma and washed platelets on the proliferation of mouse MSC cells. Mol Biol Rep 38: 2485-2490, 2011.

26 García-Martínez O, Reyes-Botella C, Díaz-Rodríguez L, De Luna-Bertos E, Ramos-Torrecillas J, Vallecillo-Capilla MF and Ruiz C: Effect of platelet-rich plasma on growth and antigenic profile of human osteoblasts and its clinical impact. J Oral Maxillofac Surg 70: 1558-1564, 2012.

27 Pappas PG, Kauffman CA, Andes DR, Clancy CJ, Marr KA, Ostrosky-Zeichner L, Reboli AC, Schuster MG, Vazquez JA, Walsh TJ, Zaoutis TE and Sobel JD: Clinical practice guideline for the management of candidiasis: 2016 update by the infectious diseases society of america. Clin Infect Dis 62: e1e50, 2016.

28 Gardner DK, Lane M, Stevens J, Schlenker T and Schoolcraft WB: Blastocyst score affects implantation and pregnancy outcome: towards a single blastocyst transfer. Fertil Steril 73: 1155-1158, 2000.

29 Coughlan C, Ledger W, Wang Q, Liu F, Demirol A, Gurgan T, Cutting R, Ong K, Sallam H and Li TC: Recurrent implantation failure: definition and management. Reprod Biomed Online 28: 14-38, 2014.

30 Salim R, Ben-Shlomo I, Colodner R, Keness Y and Shalev E: Bacterial colonization of the uterine cervix and success rate in assisted reproduction: results of a prospective survey. Hum Reprod 17: 337-340, 2002.

31 Greenhalgh DG: The role of apoptosis in wound healing. Int J Biochem Cell Biol 30: 1019-1030, 1998.

32 Sato Y, Ohshima T and Kondo T: Regulatory role of endogenous interleukin-10 in cutaneous inflammatory response of murine wound healing. Biochem Biophys Res Commun 265: 194-199, 1999.
33 Ashcroft GS, Yang X, Glick AB, Weinstein M, Letterio JJ, Mizel DE, Anzano M, Greenwell-Wild T, Wahl SM and Deng C: Mice lacking Smad3 show accelerated wound healing and an impaired local inflammatory response. Nat Cell Biol 1: 260, 1999.

34 Serhan CN: Controlling the resolution of acute inflammation: a new genus of dual anti-inflammatory and proresolving mediators. J Periodontol 79: 1520-1526, 2008.

35 Teder P, Vandivier RW, Jiang D, Liang J, Cohn L, Puré E, Henson PM and Noble PW: Resolution of lung inflammation by CD44. Science 296: 155-158, 2002.

36 Kon E, Buda R, Filardo G, Di Martino A, Timoncini A, Cenacchi A, Fornasari PM, Giannini S and Marcacci M: Plateletrich plasma: intra-articular knee injections produced favorable results on degenerative cartilage lesions. Knee Surg Sports Traumatol Arthrosc 18: 472-479, 2010.

37 Andia I and Maffulli N: Platelet-rich plasma for managing pain and inflammation in osteoarthritis. Nat Rev Rheumatol 9: 721, 2013.

38 Foster TE, Puskas BL, Mandelbaum BR, Gerhardt MB and Rodeo SA: Platelet-rich plasma: from basic science to clinical applications. Am J Sports Med 37: 2259-2272, 2009.

39 Zhu Y, Yuan M, Meng HY, Wang AY, Guo QY, Wang Y and Peng J: Basic science and clinical application of platelet-rich plasma for cartilage defects and osteoarthritis: a review. Osteoarthritis Cartilage 21: 1627-1637, 2013.

40 Gaissmaier C, Koh JL and Weise K: Growth and differentiation factors for cartilage healing and repair. Injury 39: 88-96, 2008.

41 Christgau M, Moder D, Hiller K-A, Dada A, Schmitz G and Schmalz G: Growth factors and cytokines in autologous platelet concentrate and their correlation to periodontal regeneration outcomes. J Clin Periodontol 33: 837-845, 2006.

42 Vogrin M, Rupreht M, Crnjac A, Dinevski D, Krajnc Z and Rečnik G: The effect of platelet-derived growth factors on knee stability after anterior cruciate ligament reconstruction: a prospective randomized clinical study. Wien Klin Wochenschr 122: 91-95, 2010.

43 Chang Y, Li J, Chen Y, Wei L, Yang X, Shi Y and Liang X: Autologous platelet-rich plasma promotes endometrial growth and improves pregnancy outcome during in vitro fertilization. Int J Clin Exp Med 8: 1286, 2015.

44 Hosseini L, Shirazi A, Naderi MM, Shams-Esfandabadi N, Boroujeni SB, Sarvari A, Sadeghnia S, Behzadi B and Akhondi MM: Platelet-rich plasma promotes the development of isolated human primordial and primary follicles to the preantral stage. Reprod Biomed Online 35: 343-350, 2017.

45 Marini MG, Perrini C, Esposti P, Corradetti B, Bizzaro D, Riccaboni P, Fantinato E, Urbani G, Gelati G and Cremonesi F: Effects of platelet-rich plasma in a model of bovine endometrial inflammation in vitro. Reprod Biol Endocrinol 14: $58,2016$.

46 El-Sharkawy H, Kantarci A, Deady J, Hasturk H, Liu H, Alshahat $M$ and Van Dyke TE: Platelet-rich plasma: growth factors and pro-and anti-inflammatory properties. J Periodontol 78: 661-669, 2007.

47 Alam R, Forsythe PA, Lett-Brown MA and Grant JA: Interleukin-8 and RANTES inhibit basophil histamine release induced with monocyte chemotactic and activating factor/monocyte chemoattractant peptide-1 and histamine releasing factor. Am J Respir Cell Mol Biol 7: 427-433, 1992. 
48 Sills ES, Rickers NS, Li X and Palermo GD: First data on in vitro fertilization and blastocyst formation after intraovarian injection of calcium gluconate-activated autologous platelet rich plasma. Gynecol Endocrinol 34: 756-760, 2018.

49 Pantos K, Nitsos N, Kokkali G, Vaxevanoglou T, Markomichali C, Pantou A, Grammatis M, Lazaros L and Sfakianoudis K: Ovarian rejuvenation and folliculogenesis reactivation in perimenopausal women after autologous platelet-rich plasma treatment. In: Abstracts, ESHRE 32nd Annual Meeting, pp. 3-6, 2016
50 Sfakianoudis K, Simopoulou M, Nitsos N, Rapani A, Pantou A, Vaxevanoglou T, Kokkali G, Koutsilieris M and Pantos K: A case series on platelet-rich plasma revolutionary management of poor responder patients. Gynecol Obstet Invest 22: 1-8, 2018.

Received November 18, 2018

Revised December 3, 2018

Accepted December 11, 2018 